\title{
Isquemia arterial aguda de membros superiores em pacientes diagnosticados com COVID-19: série de casos
}

\author{
Acute upper limb arterial ischemia in patients diagnosed with COVID-19: case series \\ Felipe Damascena Rosa' (D, Marcelo Calil Burihan', Elexandra Aparecida Simões', João Paulo de Souza Abdala', \\ Orlando da Costa Barros ${ }^{1}$, Felipe Nasser ${ }^{1,2}$
}

\begin{abstract}
Resumo
A infecção pelo coronavírus 2 causador da síndrome respiratória aguda grave (SARS-CoV-2) em humanos foi detectada pela primeira vez em Wuhan, na China, em 2019 e dispersada mundialmente ao longo de 2020. As diferentes manifestações clínicas, com amplo espectro de apresentação, desde infecções assintomáticas até formas graves que podem levar a óbito, são desafiadoras. Este trabalho objetiva descrever uma série de quatro casos de isquemia arterial aguda dos membros superiores em pacientes diagnosticados com COVID-19, os quais foram manejados clinicamente com anticoagulação, antiagregação plaquetária e uso de prostanoides. Dois pacientes receberam alta hospitalar com regressão e delimitação da área isquêmica, sem necessidade de intervenção cirúrgica, e dois pacientes faleceram em decorrência de complicações pulmonares. Uma adequada compreensão da fisiopatologia dessa doença pode favorecer um melhor manejo clínico de suas complicações.
\end{abstract}

Palavras-chave: COVID-19; anticoagulantes; embolia e trombose; isquemia; prostaglandinas.

\begin{abstract}
Infection by coronavirus 2, cause of the severe acute respiratory syndrome (SARS-CoV-2) in humans, was detected for the first time in Wuhan, China, in 2019, and spread globally over the course of 2020. Its different clinical manifestations are challenging, with a wide spectrum of presentations, ranging from asymptomatic infections to severe forms that can result in death. The objective of this study is to describe a series of four cases of acute arterial ischemia involving the upper limbs in patients diagnosed with COVID-19, which were managed clinically with anticoagulation, platelet antiaggregation, and prostanoids. Two patients were discharged from hospital with regression and delimitation of the ischemic zone, without needing surgical intervention, while two patients died from pulmonary complications. Adequate understanding of the pathophysiology of this disease could support better clinical management of its complications.
\end{abstract}

Keywords: COVID-19; anticoagulants; emboli and thrombosis; ischemia; prostaglandins.

Como citar: Rosa FD, Burihan MC, Simões EA, Abdala JPS, Barros OC, Nasser F. Isquemia arterial aguda de membros superiores em pacientes diagnosticados com COVID-19: série de casos. J Vasc Bras. 2021;20:e20200234. https://doi. org/10.1590/1677-5449.200234

\footnotetext{
${ }^{1}$ Hospital Santa Marcelina, São Paulo, SP, Brasil.

${ }^{2}$ Hospital Israelita Albert Einstein, São Paulo, SP, Brasil.

Fonte de financiamento: Nenhuma.

Conflito de interesse: Os autores declararam não haver conflitos de interesse que precisam ser informados.

Submetido em: Janeiro 11, 2021. Aceito em: Março 17, 2021.
}

O estudo foi realizado no Hospital Santa Marcelina, Unidade de Itaquera, São Paulo, SP, Brasil. 


\section{INTRODUÇÃO}

O surgimento de uma nova doença causada coronavírus 2 causador da síndrome respiratória aguda grave (SARS-COV-2) em dezembro de $2019 \mathrm{em}$ Wuhan, na China, que se espalhou ao redor do mundo no primeiro semestre de 2020, impôs novos desafios para a caracterização clínica e fisiopatológica dessa enfermidade. Denominada doença do novo coronavírus (COVID-19) pela Organização Mundial de Saúde, a doença foi declarada pandêmica em março de $2020^{1}$.

Inicialmente apresentada como síndrome respiratória de vias aéreas inferiores, logo se observou uma gama de sinais, sintomas e apresentações clínicas, que vão desde casos oligossintomáticos até àqueles que evoluem com insuficiência respiratória, coagulopatia, disfunção de múltiplos órgãos e morte ${ }^{2,3}$. Estudos têm apontado para um estado de hipercoagulabilidade, que poderia culminar em complicações da micro e da macrocirculação $0^{4-6}$.

A seguir, será relatada uma série de quatro pacientes que apresentaram isquemia arterial aguda dos membros superiores e diagnóstico de COVID-19. Este trabalho foi aprovado pelo Comitê de Ética em Pesquisa CAAE 37208320.5.0000.0066, número do parecer: 4.303.538.

\section{RELATO DE CASOS}

\section{Caso 1}

Paciente do sexo feminino, 18 anos de idade, previamente hígida, deu entrada no pronto-socorro com queixa de dor contínua, de moderada intensidade, progressiva, associada a cianose de quarto quirodáctilo direito, com início há 2 dias. Negava febre, trauma, queixas respiratórias, alergias, tabagismo, uso de drogas injetáveis ou contato com pessoas diagnosticadas com COVID-19. Referia fazer uso regular de anticoncepcional oral combinado (levonorgestrel $0,15 \mathrm{mg}$ e etinilestradiol $0,03 \mathrm{mg}$ ). Ao exame físico, apresentava-se sem anormalidades, exceto na palpação das extremidades superiores, em que foram observados pulsos presentes, simétricos, redução da temperatura a nível de falange distal de quarto quirodáctilo direito e cianose não fixa (Figura 1). Os exames laboratoriais à admissão estão discriminados na Tabela 1. A pesquisa do fator reumatoide e do anticorpo anti-DNA foi negativa, e do anticoagulante lúpico foi positiva. A ecografia vascular (EV) do membro superior direito demonstrou artérias pérvias, de calibre preservado, com fluxo trifásico, sem alterações às manobras para identificação de síndrome do desfiladeiro torácico (SDT). O exame de reação da transcriptase reversa seguida pela reação em cadeia da polimerase (RTPCR) para SARS-CoV-2 em amostra nasofaríngea foi positivo.

Foi realizado isolamento respiratório e iniciada antiagregação plaquetária com ácido acetilsalicílico (AAS), $100 \mathrm{mg} /$ dia, associado à anticoagulação plena com enoxaparina ( $1 \mathrm{mg} / \mathrm{kg}$ de $12 \mathrm{em} 12 \mathrm{~h})$. Devido à manutenção de queixas álgicas, associou-se alprostadil por via intravenosa ( $40 \mathrm{mcg}$ de $12 \mathrm{em} 12 \mathrm{~h}$ ) e prednisona em dose anti-inflamatória $(0,5 \mathrm{mg} / \mathrm{kg} / \mathrm{dia})$ por 7 dias.

Tabela 1. Exames laboratoriais à admissão.

\begin{tabular}{|c|c|c|c|c|}
\hline & Paciente 1 & Paciente 2 & Paciente 3 & Paciente 4 \\
\hline Hemoglobina (g/dL) & 10,3 & 10,7 & 12,4 & 10,7 \\
\hline Hematócrito (\%) & 30,9 & 32,1 & 36,2 & 31 \\
\hline Leucócitos/mL & 5.906 & 9.858 & 13.680 & 4.278 \\
\hline Plaquetas $/ \mathrm{mL}$ & 229.400 & 218.800 & 222.063 & 181.500 \\
\hline RNI & 1,08 & 1,37 & 1,2 & 1,32 \\
\hline rTTPA & 1,04 & 1,03 & 1,08 & 1,26 \\
\hline PCR (mg/dL) & 11 & 15,9 & 35,3 & 115,2 \\
\hline CPK & 35 & 65 & 260 & 106 \\
\hline Ureia $(\mathrm{g} / \mathrm{dL})$ & 28 & 50 & 39 & 90 \\
\hline Creatinina $(\mathrm{g} / \mathrm{dL})$ & 0,52 & 1,24 & 0,6 & 1,41 \\
\hline D-dímero (mcg/dL) & 1.750 & - & 1.825 & 881 \\
\hline Glicemia (mg/dL) & 75 & 102 & 136 & 112 \\
\hline HB Glicada (\%/dL) & 4,8 & - & 10,8 & - \\
\hline Sódio (mg/dL) & 131 & 142 & 141 & 138 \\
\hline Potássio (mg/dL) & 4,1 & 3,6 & 3,9 & 5,1 \\
\hline Cálcio (mg/dL) & 9,4 & 8,4 & 8,9 & 8,5 \\
\hline Magnésio (mg/dL) & 2,2 & 1,9 & 1,95 & 1,81 \\
\hline
\end{tabular}

RNI = razão de normatização internacional; rTTPA = relação de tempo de tromboplastina parcial ativada; PCR = reação em cadeia da polimerase; CPK = creatinofosfoquinase; $\mathrm{HB}=$ hemoglobina 


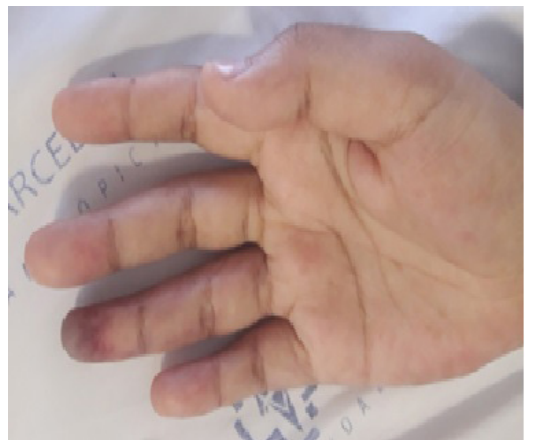

Figura 1. Cianose em quarto quirodáctilo direito.

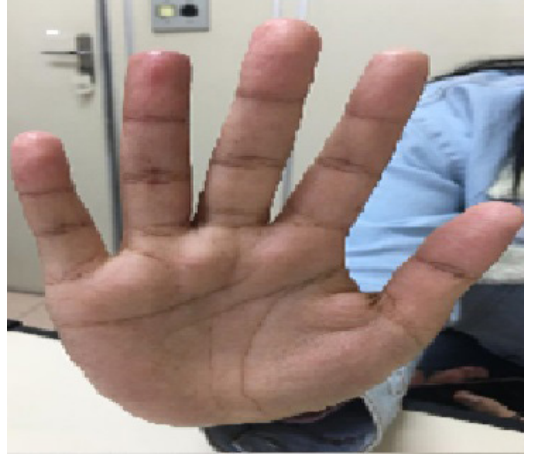

Figura 2. Quarto quirodáctilo direito após 14 dias.

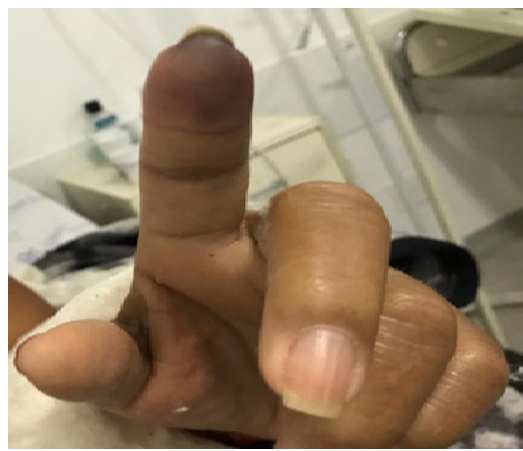

Figura 3. Cianose fixa em segundo quirodáctilo direito.

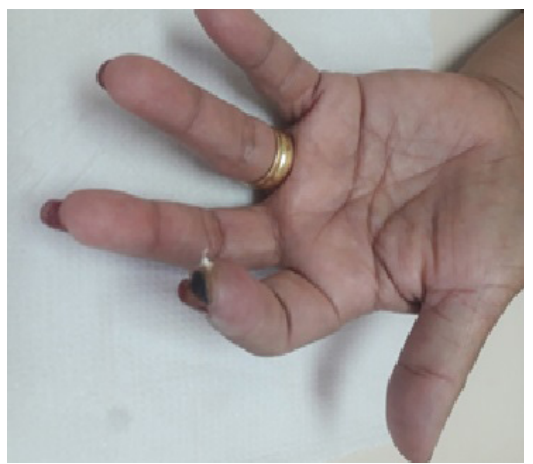

Figura 4. Necrose seca, delimitada, segundo quirodáctilo direito após 14 dias.
A paciente evoluiu com melhora da dor, regressão da cianose e do gradiente térmico, recebendo alta hospitalar no $14^{\circ}$ dia de internação (Figura 2).

\section{Caso 2}

Paciente do sexo feminino, 57 anos de idade, portadora de hipertensão arterial sistêmica (HAS) e diabetes melito (DM) tipo 2, deu entrada no prontosocorro com relato de dor intensa e contínua em segundo quirodáctilo esquerdo, não responsiva a analgésicos comuns, com início há 2 dias, associado à parestesia, redução de temperatura e cianose fixa (Figura 3). Negava febre, trauma, tosse, uso de drogas injetáveis ou tabagismo. Relatava fazer uso regular de glibenclamida, enalapril, AAS, cilostazol e nifedipina. Ao exame físico geral, não foram detectadas anormalidades; a palpação das extremidades superiores e inferiores identificou pulsos presentes, simétricos, com redução de temperatura a nível da falange distal de segundo quirodáctilo esquerdo. Os exames laboratoriais à admissão estão discriminados na Tabela 1. A EV do membro superior esquerdo demonstrou artérias pérvias, de calibre preservado, com fluxo trifásico, sem estenoses significativas e sem alterações às manobras para identificação de SDT. O ecocardiograma apresentou fração de ejeção preservada, sem áreas de adinamia ventricular ou trombos identificáveis em câmaras cardíacas.

Optou-se por anticoagulação plena com enoxaparina $(1 \mathrm{mg} / \mathrm{kg}$ de $12 \mathrm{em} 12 \mathrm{~h}$ ), associado a alprostadil por via intravenosa (40 mcg de $12 \mathrm{em} 12 \mathrm{~h}$ ) e prednisona em dose anti-inflamatória $(0,5 \mathrm{mg} / \mathrm{kg} / \mathrm{dia})$ por 7 dias. A paciente evoluiu bem; no entanto, devido à manutenção de cianose fixa de extremidade, optouse por manter a anticoagulação oral com varfarina, objetivando uma razão de normatização internacional (RNI) entre 2 e 3, a fim de acompanhar a delimitação do processo ambulatorialmente. A paciente recebeu alta no $7^{\circ}$ dia de internação, com varfarina oral e RNI de 2,15. No retorno ambulatorial em 14 dias, foi observada pequena necrose seca em falange distal (Figura 4). Como não foi realizada coleta de $s w a b$ nasal durante a internação, solicitou-se sorologia para COVID-19, sendo detectado IgG positivo.

\section{Caso 3}

Paciente do sexo feminino, 75 anos de idade, portadora de obesidade grau III, DM tipo 2, HAS, ex-tabagista (40 maços-ano), com sequela motora em membro inferior esquerdo devido a um acidente vascular encefálico isquêmico prévio. Compareceu ao pronto-socorro queixando-se de dor há 7 dias em mão esquerda, de início súbito, contínua, com piora progressiva e evolução para cianose de quirodáctilos. 
Negava trauma, febre, tosse, dispneia ou uso de drogas injetáveis. Relatava uso de metformina, glibenclamida, insulina, atenolol, hidroclorotiazida, fenitoína e AAS. Ao exame físico, apresentava-se com pulsos axilar, braquial e ulnar presentes e normais e pulso radial ausente, cianose fixa e redução da temperatura do terceiro quirodáctilo e cianose não fixa dos demais quirodáctilos à esquerda (Figura 5). Os exames laboratoriais à admissão estão discriminados na Tabela 1. A EV do membro superior esquerdo evidenciou artérias pérvias, de calibre preservado, com fluxo trifásico, com aterosclerose difusa e oclusão aterosclerótica da artéria radial em seu terço proximal, e as manobras para identificação de SDT foram negativas. O ecocardiograma apresentou fração de ejeção ventricular de $61 \%$, sem áreas de adinamia ventricular ou trombos identificáveis em câmaras cardíacas. A tomografia computadorizada (TC) de tórax evidenciou imagens em vidro fosco difusamente, comprometendo mais que $50 \%$ dos campos pulmonares bilateralmente (Figura 6). O RT-PCR para SARS-CoV-2 foi positivo.

Optou-se por iniciar anticoagulação plena com enoxaparina ( $1 \mathrm{mg} / \mathrm{kg}$ de $12 \mathrm{em} 12 \mathrm{~h}$ ), AAS $100 \mathrm{mg} / \mathrm{dia}$, associado a alprostadil por via intravenosa $(40 \mathrm{mcg}$ de $12 \mathrm{em} \mathrm{12h).} \mathrm{A} \mathrm{paciente} \mathrm{evoluiu} \mathrm{com} \mathrm{quadro}$ respiratório grave e óbito após 27 dias de internação.

\section{Caso 4}

Paciente do sexo feminino, 84 anos de idade, deu entrada no pronto-socorro com história de tosse seca, mal-estar geral, inapetência e episódios recorrentes de dispneia há 7 dias. A paciente relatou os seguintes antecedentes pessoais: HAS e dislipidemia, em uso de losartana, AAS e sinvastatina. Ao exame físico, apresentava crepitações difusas em todo o hemitórax direito e terço inferior de hemitórax esquerdo, frequência respiratória de 24 incursões respiratórias por minuto (irpm), saturação de oxigênio de $77 \%$ em ar ambiente e de $92 \%$ com máscara de oxigênio (4 L/min). $\mathrm{Na}$ palpação das extremidades superiores e inferiores, foram detectados pulsos presentes, simétricos, sem gradiente térmico. Os exames laboratoriais à admissão estão discriminados na Tabela 1. Foi realizado RT-PCR de amostra nasofaríngea, sendo positivo para SARS-CoV-2. A TC de tórax revelou opacidades interstício-alveolares em padrão vidro fosco difusamente (Figura 7).

A paciente evoluiu com gravidade, e, no $10^{\circ}$ dia de internação em unidade de terapia intensiva (UTI), apresentou cianose fixa somente em mão direita, com pulsos presentes (Figura 8). Foi iniciado protocolo de anticoagulação plena com heparina não fracionada, com contenção da isquemia. No entanto, a paciente

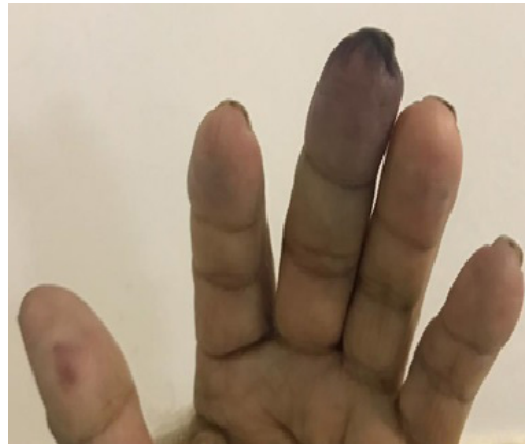

Figura 5. Cianose fixa de terceiro quirodáctilo esquerdo.

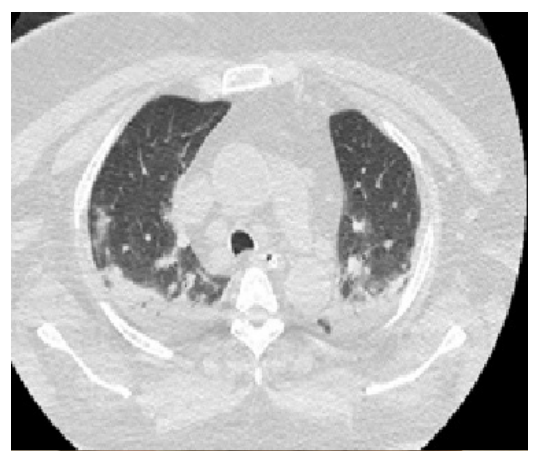

Figura 6. Tomografia computadorizada, corte axial de tórax, com imagem em vidro fosco bilateralmente.

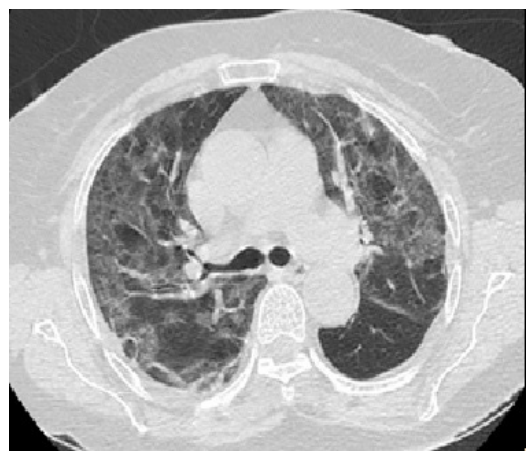

Figura 7. Tomografia computadorizada, corte axial de tórax, com imagem em vidro fosco bilateralmente.

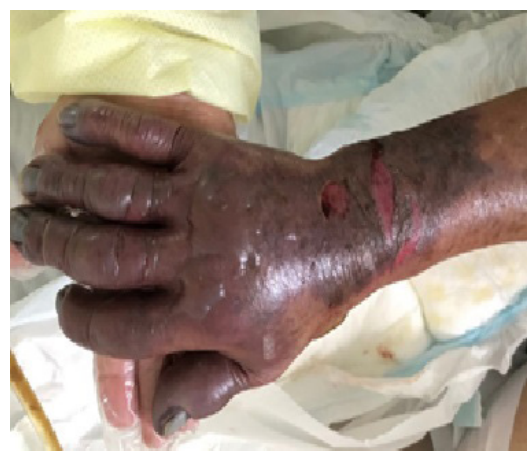

Figura 8. Mão direita com cianose fixa, lesões bolhosas e pulsos distais presentes. 
foi a óbito por complicações infecciosas e pulmonares após 42 dias de internação.

\section{DISCUSSÃO}

A apresentação inicial da COVID-19 é usualmente com sintomas respiratórios, como tosse seca, dor de garganta, e sintomas gerais inespecíficos, como adinamia, dores no corpo e febre ${ }^{2,3}$. As formas mais graves, com necessidade de intubação orotraqueal, choque séptico e coagulopatia, ocorrem geralmente em uma fase mais tardia, entre o $7^{\circ}$ e $12^{\circ}$ dia de doença, levando à necessidade de suporte intensivo ${ }^{2,3}$. A idade maior que 60 anos e a presença de comorbidades, como obesidade, DM e HAS, são reconhecidos fatores de risco para prognóstico desfavorável ${ }^{2,3}$.

Os três primeiros casos apresentados não desenvolveram queixas clínicas típicas e procuraram assistência médica devido a quadro de isquemia de microcirculação dos membros superiores. As pacientes 1 e 2 tiveram evolução favorável, sem necessidade de intervenções e internação prolongada; o tratamento anticoagulante e antiagregante plaquetário foi iniciado precocemente, o que pode ter contribuído para impedir a progressão da lesão isquêmica ${ }^{7}$.

Tang et al. ${ }^{4}$ demonstraram que elevação de produtos da degradação da fibrina, entre eles o D-dímero, e alterações do sistema fibrinolítico podem estar presentes desde as fases iniciais da COVID-19, predispondo a estados pró-trombóticos e pior prognóstico ${ }^{5,6}$. Estudos anatomopatológicos identificaram deposição de fibrina nos alvéolos e espaços intersticiais dos pulmões, com evidências adicionais de trombose da microcirculação ${ }^{8}$. Por outro lado, a adoção de protocolos de anticoagulação foi associada à diminuição da mortalidade em pacientes com COVID-19,10. A presença de anticorpo anticoagulante lúpico tem sido observada em pacientes com COVID-19, e investigase sua possível relação com estado pró-trombótico ${ }^{11}$. Ainda é incerto que as alterações na hemostasia sejam devido ao efeito direto do SARS-COV-2 ou se seria consequência da resposta inflamatória exacerbada, a chamada "tempestade de citoninas"12. Nesse sentido, o uso da heparina poderia contribuir tanto pelo seu efeito anticoagulante quanto pelo seu efeito anti-inflamatório ${ }^{13-17}$.

O uso de prostanoides em casos de isquemia crítica sem condições de revascularização é descrito na literatura, havendo relatos prévios de bons resultados na melhora da dor e pequeno efeito favorável na cicatrização de feridas. Além disso, há relato de caso de seu uso no contexto da COVID-19 ${ }^{18,19}$. Nos casos apresentados, foi observado melhora das queixas álgicas após a introdução de alprostadil, sem reações adversas.
$\mathrm{O}$ aumento de casos de isquemia arterial aguda tem sido relatado durante esta pandemia. Bellosta et al. ${ }^{20}$ descreveram 20 casos de isquemia arterial aguda de membros inferiores, com necessidade de revascularização cirúrgica. A mortalidade foi de $40 \%$, e a anticoagulação plena no pós-operatório foi relacionada a melhor prognóstico e menor necessidade de reintervenção no pós-operatório ${ }^{20}$. Nesta casuística, não se observaram eventos hemorrágicos maiores nos pacientes submetidos à anticoagulação, tendo aparentemente bom desfecho em relação à contenção dos fenômenos isquêmicos.

\section{REFERÊNCIAS}

1. World Health Organization [site da Internet]. Coronavirus disease (COVID-19). 2020 [atualizado 2020 nov 26; citado 2020 dez 3]. https://www.who.int/emergencies/diseases/novel-coronavirus-2019/ advice-for-public.

2. Huang $C$, Wang $Y$, Li $X$, et al. Clinical features of patients infected with 2019 novel coronavirus in Wuhan, China. Lancet. 2020;395(10223):497-506. http://dx.doi.org/10.1016/S01406736(20)30183-5. PMid:31986264.

3. Zhu N, Zhang D, Wang W, et al. A novel coronavirus from patients with pneumonia in China, 2019. N Engl J Med. 2020;382(8):727-33. http://dx.doi.org/10.1056/NEJMoa2001017. PMid:31978945.

4. Tang N, Li D, Wang X, Sun Z. Abnormal coagulation parameters are associated with poor prognosis in patients with novel coronavirus pneumonia. J Thromb Haemost. 2020;18(4):844-7. http://dx.doi. org/10.1111/jth.14768. PMid:32073213.

5. Han H, Yang L, Liu R, et al. Prominent changes in blood coagulation of patients with SARS-CoV-2 infection. Clin Chem Lab Med. 2020;58(7):1116-20. http://dx.doi.org/10.1515/cclm-2020-0188. PMid:32172226.

6. Zhou F, Yu T, Du R, et al. Clinical course and risk factors for mortality of adult inpatients with COVID-19 in Wuhan, China: a retrospective cohort study. Lancet. 2020;320(10229):1054-62. http://dx.doi.org/10.1016/S0140-6736(20)30566-3. PMid:32171076.

7. Liu X, Li Z, Liu S, et al. Potential therapeutic effects of dipyridamole in the severely ill patients with COVID-19. Acta Pharm Sin B. 2020;10(7):1205-15. http://dx.doi.org/10.1016/j.apsb.2020.04.008. PMid:32318327.

8. Magro C, Mulvey JJ, Berlin D, et al. Complement associated microvascular injury and thrombosis in the pathogenesis of severe COVID-19 infection: a report of five cases. Trans. Res. 2020;220:113. http://dx.doi.org/10.1016/j.trsl.2020.04.007. PMid:32299776.

9. Tang N, Bai H, Chen X, Gong J, Li D, Sun Z. Anticoagulant treatment is associated with decreased mortality in severe coronavirus disease 2019 patients with coagulopathy. J Thromb Haemost. 2020;18(5):1094-9. http://dx.doi.org/10.1111/jth.14817. PMid:32220112.

10. Paranjpe I, Fuster V, Lala A, et al. Association of treatment dose anticoagulation with in-hospital survival among hospitalized patients with COVID-19. J Am Coll Cardiol. 2020;76(1):122-4. http://dx.doi.org/10.1016/j.jacc.2020.05.001. PMid:32387623.

11. Bowles L, Platton S, Yartey N, et al. Lupus anticoagulant and abnormal coagulant tests in patients with COVID-19. N Engl J Med. 2020;383(3):288-90. http://dx.doi.org/10.1056/NEJMc2013656. PMid:32369280.

12. Mehta P, McAuley DF, Brown M, Sanchez E, Tattersall RS, Manson JJ. COVID-19: consider cytokine storm syndromes and 
immunosuppression. Lancet. 2020;395(10229):1033-4. http:// dx.doi.org/10.1016/S0140-6736(20)30628-0. PMid:32192578.

13. Colling ME, Kanthi Y. COVID-19-associated coagulopathy: an exploration of mechanisms. Vasc Med. 2020;25(5):471-8. http:// dx.doi.org/10.1177/1358863X20932640. PMid:32558620.

14. Li X, Liu Y, Wang L, Li Z, Ma X. Unfractionated heparin attenuates LPS-induced IL-8 secretion via PI3K/Akt/NF-kB signaling pathway in human endothelial cells. Immunobiology. 2015;220(3):399-405. http://dx.doi.org/10.1016/j.imbio.2014.10.008. PMid:25454806.

15. Shastri MD, Stewart N, Horne J, et al. In-vitro suppression of IL-6 and IL-8 release from human pulmonary epithelial cells by nonanticoagulant fraction of enoxaparin. PLoS One. 2015;10(5):e0126763. http://dx.doi.org/10.1371/journal.pone.0126763. PMid:25961885.

16. Menezes-Rodrigues FS, Tavares JGP, Oliveira MP, et al. Anticoagulant and antiarrhythmic effects of heparin in the treatment of COVID-19 patients. J Thromb Haemost. 2020;18(8):2073-5. http://dx.doi. org/10.1111/jth.14902. PMid:32408391.

17. Bikdeli B, Madhavan MV, Jimenez D, et al. COVID-19 and thrombotic or thromboembolic disease: implications for prevention, antithrombotic therapy, and follow-Up: JACC state-of-the-art review. J Am Coll Cardiol. 2020;75(23):2950-73. http://dx.doi. org/10.1016/j.jacc.2020.04.031. PMid:32311448.

18. Meini S, Dentali F, Melillo E, de Donato G, Mumoli N, Mazzone A. Prostanoids for critical limb ischemia: a clinical review and consideration of current guideline recommendations. Angiology. 2020;71(3):226-34. http://dx.doi.org/10.1177/0003319719889273. PMid:31769315.

19. Manhanelli M Fo, Duarte EJr, Mariuba J, et al. Alprostadil associated with low molecular weight heparin to treat limb ischemia caused by SARS-CoV2. J Vasc Bras. 2020;19:e20200072. http://dx.doi. org/10.1590/1677-5449.200072.

20. Bellosta R, Luzzani L, Natalini G, et al. Acute limb ischemia in patients with COVID-19 pneumonia. J Vasc Surg. 2020;72(6):186472. http://dx.doi.org/10.1016/j.jvs.2020.04.483. PMid:32360679.
Correspondência Felipe Damascena Rosa Hospital Santa Marcelina

Rua Santa Marcelina, 177, Serviço de Cirurgia Vascular e Endovascular 2-A CEP 08270-070 - São Paulo (SP), Brasil Tel.: (11) 2070-6486 E-mail: felipedcena@gmail.com

Informações sobre os autores FDR - Mestre em Medicina, Faculdade de Medicina de Botucatu, Universidade Estadual Paulista (FMB/Unesp); Cirurgião Vascular e Endovascular, Serviço de Cirurgia Vascular e Endovascular, Hospital Santa Marcelina.

MCB - Mestre em Anatomia, Universidade Federal de São Paulo (Unifesp); Cirurgião Vascular e Endovascular, Serviço de Cirurgia Vascular e Endovascular, Hospital Santa Marcelina.

EAS e JPSA - Residentes de Cirurgia Vascular, Serviço de Cirurgia Vascular e Endovascular, Hospital Santa Marcelina.

OCB - Especialista em Cirurgia Vascular, Serviço de Cirurgia Vascular e Endovascular, Hospital Santa Marcelina.

FN - Doutor em Radiologia, Universidade de São Paulo (USP);

Cirurgião Vascular e Endovascular; Supervisor, Serviço de Cirurgia Vascular e Endovascular, Hospital Santa Marcelina; Coordenador, Setor de Radiologia Vascular Intervencionista, Hospital Israelita Albert Einstein.

Contribuições dos autores Concepção e desenho do estudo: FDR, MCB, FN Análise e interpretação dos dados: FDR, MCB, EAS, JPSA, OCB, FN Coleta de dados: EAS, JPSA Redação do artigo: FDR, MCB, EAS, JPSA, OCB, FN Revisão crítica do texto: $M C B, F N$ Aprovação final do artigo: FDR, MCB, EAS, JPSA, OCB, FN Análise estatística: $M C B, F N$

Responsabilidade geral pelo estudo: FDR, MCB, EAS, JPSA, OCB, FN

*Todos os autores leram e aprovaram a versão final submetida ao J Vasc Bras. 